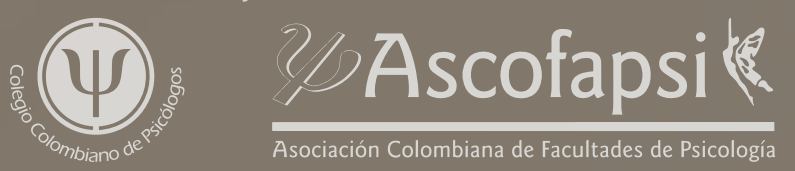

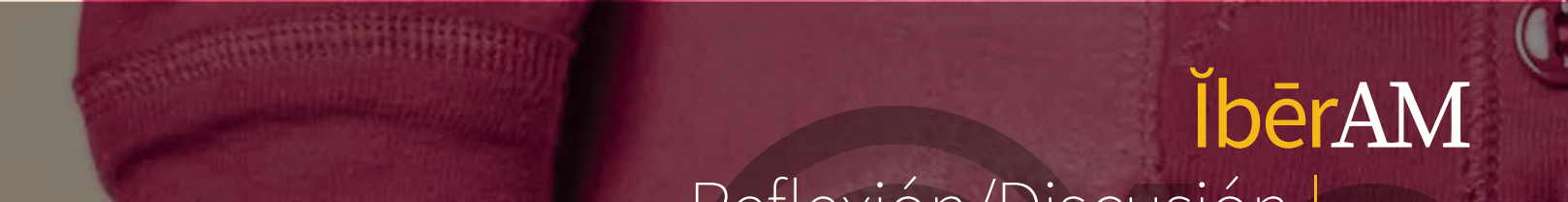
Reflexión/Discusión | Relection

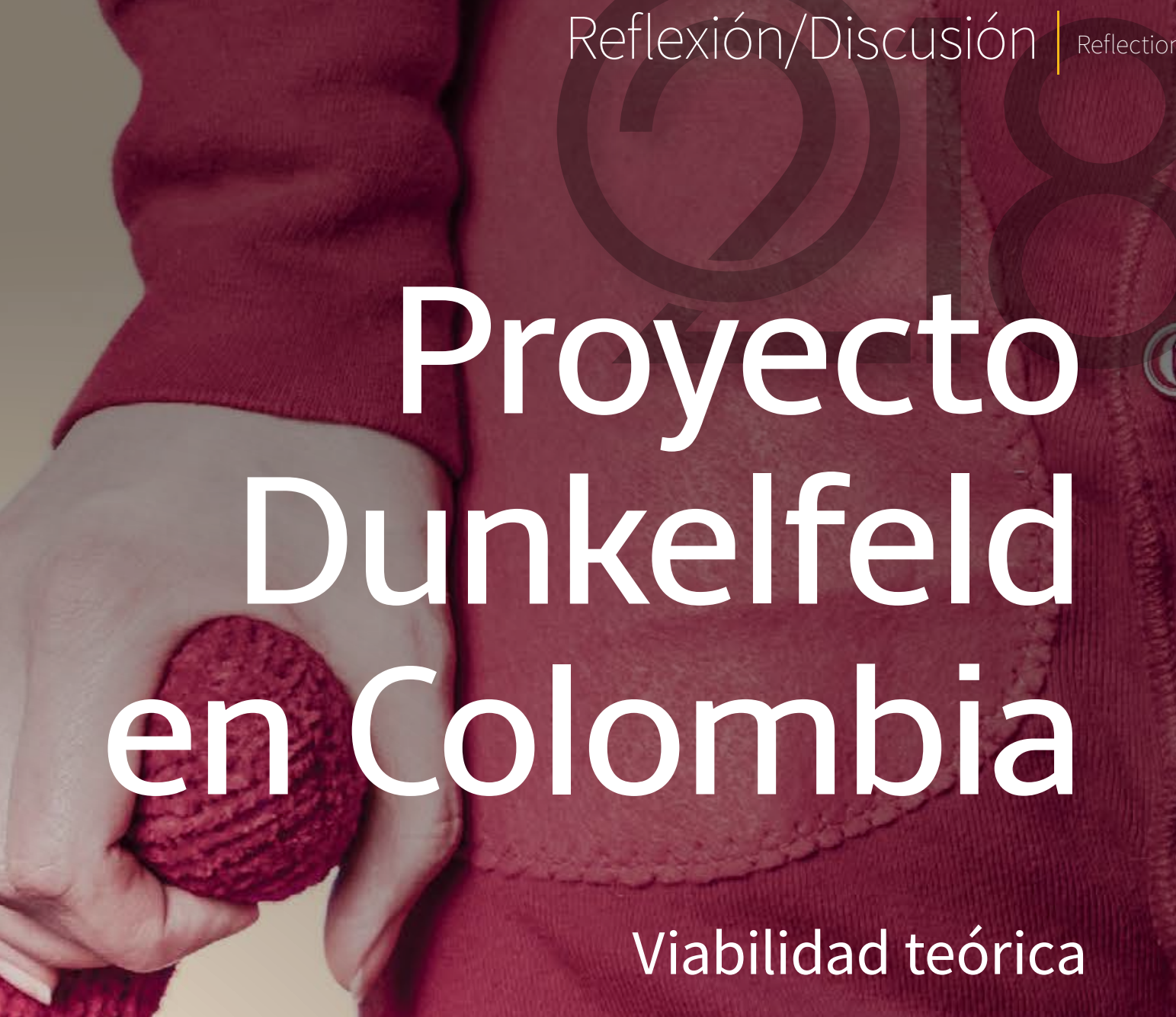

Viabilidad teórica

Santiago Amaya Nassar

Santiago Hernández Cabiedes

Dunklefeld project in Colombia: theroretical feasibility

(c)

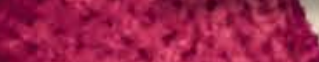

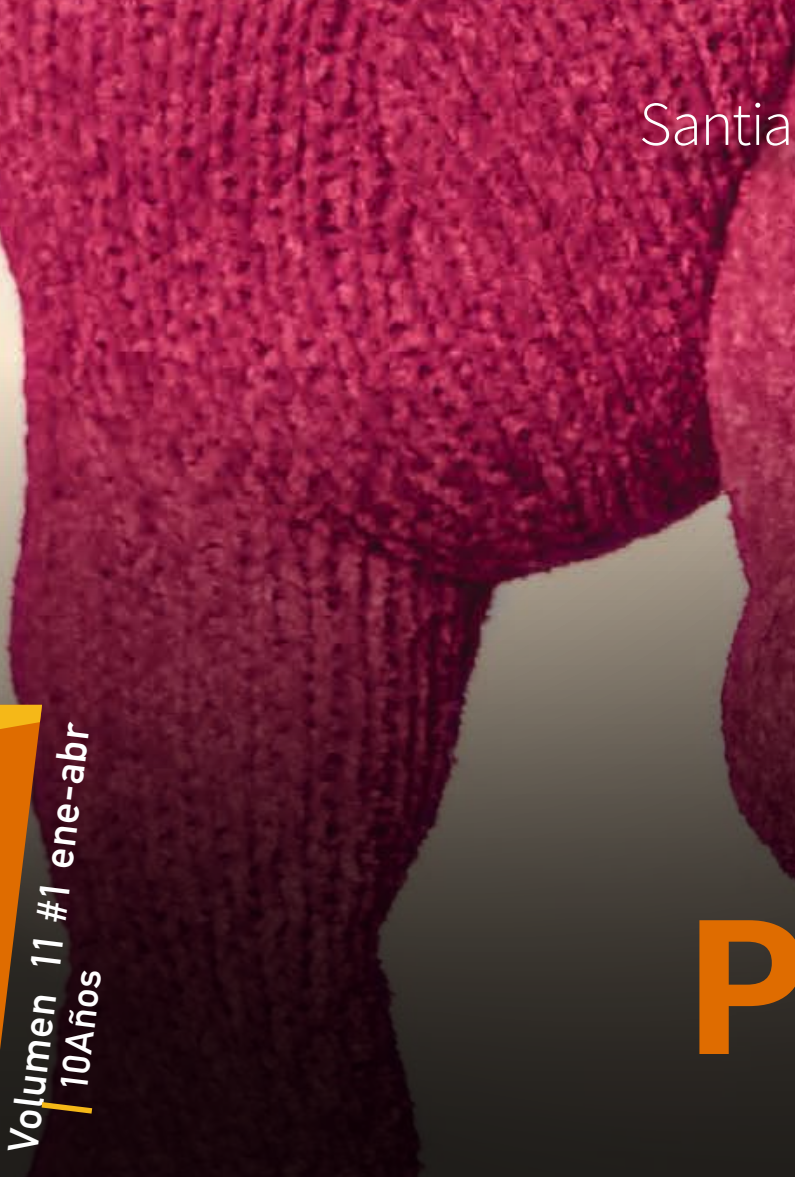

Revista Iberoamericana de

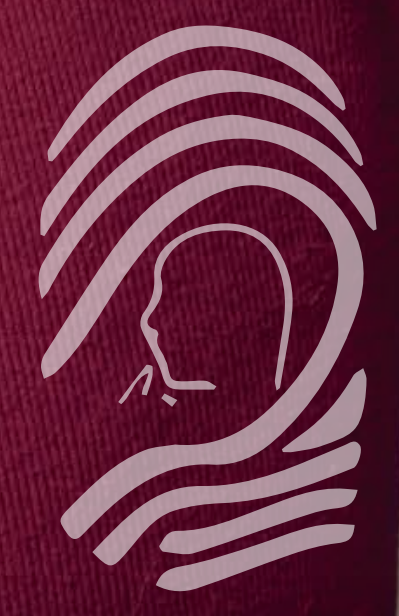

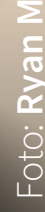
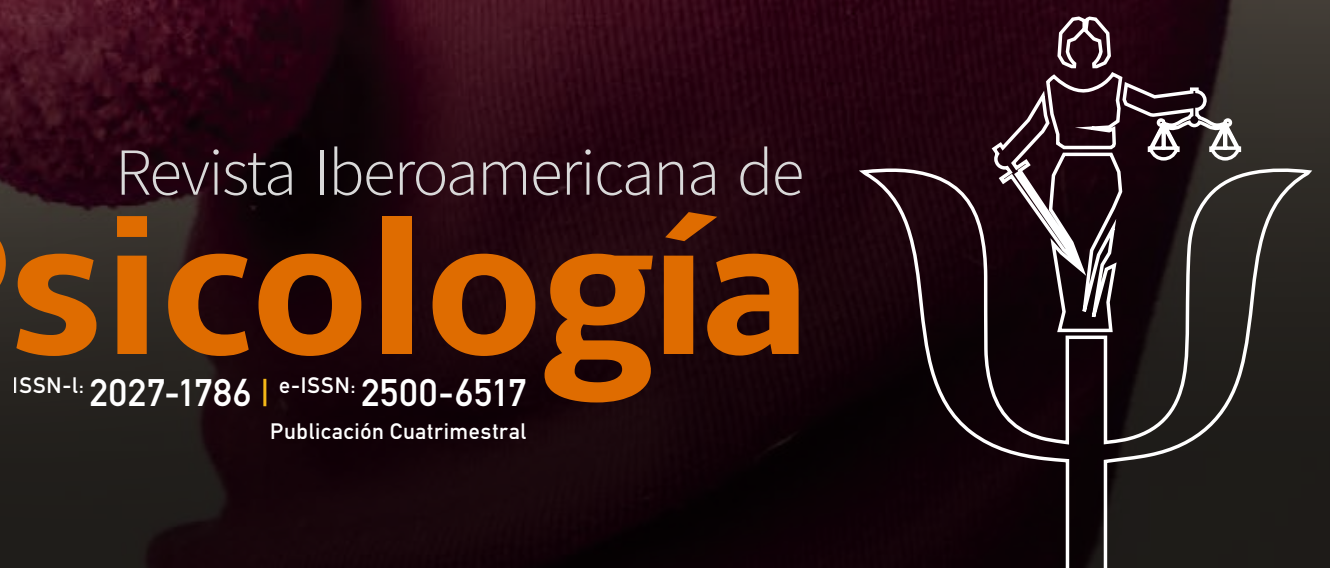
Revista Iberoamericana de

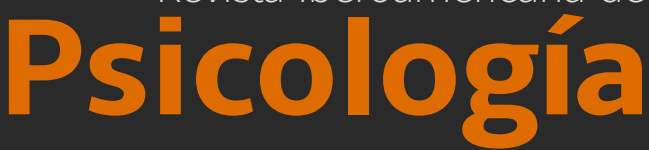

ISSN-I: 2027-1786 | e-ISSN: 2500-6517 Publicación Cuatrimestral

ID:

\section{7-1786.rip.11109}

Title: Dunklefeld project in Colombia

Subtitle: Theroretical feasibility

Título: Proyecto Dunkelfeld en Colombia

Subtítulo: Viabilidad teórica

Alt Title / Título alternativo:

[es]: Viabilidad teórica del Proyecto Dunkelfeld en Colombia

Author (s) / Autor (es):

\section{Amaya Nassar, \& Hernández Cabiedes}

Keywords / Palabras Clave:

[en]:

dunkelfeld project: child sexual abuse; prevention; pedophilia; aggressor

[es]: proyecto dunkelfeld; abuso sexual infantil; prevención; pedofilia; agresor

Submited: 2017-11-09

Acepted: 2018-03-09

\section{Resumen}

El presente es un texto reflexivo en el cual se busca explorar, de manera teórica, la viabilidad de aplicar el Proyecto Dunkelfeld en Colombia. Dicho proyecto es una estrategia creada en Alemania en la que pedófilos y agresores sexuales infantiles brindan atención primaria, lo anterior con el fin de reducir el abuso sexual infantil (ASI). Primero se expone la problemática del $\boldsymbol{A} \boldsymbol{S I}$, así como la necesidad de un programa integral que tome en consideración a los agresores. Acto seguido, se presenta el Proyecto Dunkelfeld tomando en cuenta el abordaje y los alcances obtenidos. Una vez presentado el proyecto, se consideran los abordajes clínicos, jurídicos,

forenses psicológicos y psiquiátricos, al

igual que la necesidad de apoyo de parte los medios de comunicación para poder desarrollar efectivamente el programa en Colombia. Se concluye que el programa puede ser viable en el país, sin embargo, requiere de un gran apoyo interinstitucional e interprofesional. También resulta necesario aclarar a la población general que el proyecto no es una apología al ASI o a la pedofilia, sino que busca reducir el abuso de niños, niñas y adolescentes.

Santiago Amaya Nassar, MSc Psi

ORCID: $\quad$ 0000-0001-7768-9043

Source I Filiacion:

Asociación Colombiana de Criminología (ACC)

BIO:

Psicólogo de la Pontificia Universidad Javeriana, Máster en evaluación psicológica

forense y penitenciaria. Director académico

Asociación Colombiana de Criminología (ACC).

City I Ciudad:

Bogotá DC [co]

e-mail:

santiagoamaya@acc.org.co

\section{Abstract}

This article is a reflexive text that explores theoretically the viability of applying the Dunkelfeld Project in Colombia. Such project is a strategy created in Germany in which pedophiles and sexual aggressors provide primary attention with the aim of reducing child sexual abuse (CSA). First, the problem of CSA is presented, along with the need for an integrated program that considers and addresses the aggressors. Second, the Dunkelfeld project is presented taking into account the approach and achievements of the program. Once the project is presented, we address the psychological and psychiatrical approaches (clinical, forensic and legal) of, as well as the need for support from of the media to be able to effectively develop the program in Colombia. In conclusion the program may be viable in the country, nevertheless, a great

inter-institutional and inter-professional support is needed. Also, it must be clarified to the general population that the program is not an apology of CSA or pedophilia, on the contrary, it seeks to reduce teen and child abuse.

Santiago Hernández Cabiedes, MSc Psi

Source I Filiacion:

Independiente

BIO:

Psicólogo de la Pontificia Universidad Javeriana, Máster en evaluación psicológica forense y penitenciaria

City I Ciudad:

Bogotá DC [co]

e-mail:

hernandezcabiedesantiago@gmail.com

Citar como:

Amaya Nassar, S., \& Hernández Cabiedes, S. (2018). Proyecto Dunkelfeld en Colombia: Viabilidad teórica. Revista Iberoamericana de Psicología issn-l:2027-1786, 11 (1), 91-100.

Obtenido de: https://revistas.iberoamericana.edu.co/index.php/ripsicologia/article/view/1292 


\title{
Proyecto Dunkelfeld en Colombia Viabilidad teórica
}

\author{
Dunklefeld project in Colombia: Theroretical feasibility \\ Santiago Amaya Nassar
Santiago Hernández Cabiedes
}

\section{Introducción}

En la última década en Colombia el Abuso Sexual Infantil (ASI) se ha constituido en un fenómeno de gran impacto en sociedad, no sólo por la población víctima (niños y adolescentes todos menores edad) sino por las circunstancias y contextos en los que toma lugar, pues la mayoría de los casos suele presentarse al interior de las familias. Lo anterior hace que se considere una problemática de salud y seguridad que requiere una intervención oportuna de parte de la sociedad, del Estado y sus instituciones, así como de las organizaciones no gubernamentales y la academia, entre otros (Rodríguez, 2003; Vélez, Henao, Ordoñez, \& Gómez, 2015; Uribe, 2011).

En aras de atender y mitigar dicha problemática se han creado programas a cargo de instituciones como el Instituto Colombiano de Bienestar Familiar (ICBF) y la Fiscalía. Sin embargo, al día de hoy no se ha logrado un gran impacto pues las cifras de denuncias por $\boldsymbol{A S I}$ no han disminuido considerablemente y la impunidad sigue presente (INMLYCF, 2016; 2017);. Evidencia de lo anterior se halla en los informes de medicina legal que indican un incremento del $\mathbf{1 1} \%$ en casos de violencia contra las mujeres, en el periodo de enero 2016 a octubre de 2017. La mayoría de las víctimas en estos casos citados eran niñas de 05-09 años y de 10-14 años, lo cual es congruente con un aumento en las denuncias de abuso sexual en estas poblaciones (INMLYCF, 2017).

Una de las grandes dificultades que se enfrentan en este contexto es la falta de agilidad en los procesos y/o, en algunos casos, la revictimización (Marroquín, 2016; Rodríguez, 2003); En la actualidad el sistema de justicia colombiano se encuentra bajo una sobredemanda que le impide operar correctamente y valorar los casos con la minucia necesaria para hacer un mejor ejercicio de la justicia. A lo anterior hay que agregar la falta de conocimiento de parte de los operadores judiciales sobre las implicaciones psicológicas que acarrea este fenómeno a las partes involucradas (Uribe, 2011; Calderón, 2016; Britto, 2009; Caracol Radio, 2011).

Sin embargo resulta preciso mencionar que los programas de intervención en sí también adolecen de fallas, las cuales radican principalmente en su enfoque binario, pues la mayoría de ellos abordan el fenómeno concibiendo sólo dos partes: los menores en tanto potenciales víctimas (Villanueva, 2005; Unicef, 2017; González \& González, 2004) y sus entornos, obviando al victimario y sus características psicológicas. Así pues, se lleva a cabo un abordaje pedagógico y didáctico mediante el cual se busca, principalmente, crear consciencia en los niños, niñas y adolescentes, sobre su desarrollo físico y psicológico, y sobre la apropiación de sus derechos. Otro eje fundamental de estos programas es el trabajo con los adultos que componen los círculos sociales más cercanos a los menores, a fin de crear espacios de protección y vigilancia (Vélez, Henao, Ordoñez, \& Gómez, 2015; Te protejo, 2018; Castellanos, Olmos, \& Hernández, 2016; Goicoechea, Nañez, \& Molino, 2001; ICBF, 2012).

A lo anterior hay que sumar el hecho que en Colombia no existe una cultura de salud mental que considere la existencia de variaciones en la personalidad, la sexualidad y en el control de impulsos, las cuales explican que algunas personas desarrollen una mayor tendencia a incurrir en actos desaprobados socialmente que pueden terminar en hechos desafortunados como una agresión sexual a un menor (Ministerio de Salud, 2015; Rubiano, 2017).

Teniendo en cuenta lo mencionado hasta el momento, resulta útil y necesario hacer énfasis en esos factores de personalidad y sexualidad a la hora de diseñar y crear programas asertivos de prevención y contención, dirigidos a aquellas personas quienes aún no son conscientes que su condición no es una tendencia delictiva, sino el resultado de unos procesos psicológicos que se traducen en una psicopatología, tal como lo son las parafilias.

Así entonces, la psicología entra a jugar un rol fundamental en la atención y abordaje del $\boldsymbol{A S I}$, especialmente en el campo de acción forense donde se evalúan presuntos casos y se determina si un agresor presenta o no los rasgos psicológicos de una persona con una parafilia como la pedofilia. Para llevar a cabo lo anterior se recurre a varias herramientas de evaluación y, principalmente, se adoptan teorías que explican los factores de riesgo y protección, y que exploran las condiciones psicológicas. Dichas teorías son la lente a través de la cual se ha de brindar una nueva mirada al fenómeno en cuestión para así 


\section{Proyecto Dunkelfeld en Colombia}

\section{Viabilidad teórica}

abordarsu prevención, considerando la existencia de ciertas tendencias que escapan el control del individuo y que lo llevan a incurrir en un abuso que no sólo afecta a la víctima y a la sociedad, sino en muchos casos al victimario mismo quien, en ocasiones, por desconocimiento u omisión no atiende esas conductas a tiempo.

\section{Marco de Referencia}

Para las evaluaciones y análisis la psicología forense trabaja con teorías de tipologías de agresores sexuales infantiles y pedófilos, las cuales, en su mayoría, clasifican los pedófilos como: primarios (o fijados) y secundarios (o regresivos) (Nicholas Groth, 1978 citado por Arias \& Azor, 2009; Vázquez, 2007 citado por Arias \& Azor, 2009; Bieber, 2012; Oliverio \& Graziosi, 2004 citados por Arias \& Azor, 2009; Arias \& Azor, 2009). Los primarios o fijados son personas quienes sienten una atracción sexual exclusiva hacia niños y rara vez sienten culpa por sus acciones. Por su parte, los regresivos o secundarios sienten atracción sexual por personas adultas o de su misma edad, pero tienen poco control de impulsos y, debido a esto, en algunas ocasiones pueden tener conductas sexuales desviadas que terminen en casos de $\boldsymbol{A S I}$ (Nicholas Groth, 1978 citado por Arias \& Azor, 2009; Bieber, 2012; Becerra-García \& García-León, 2012). Es preciso tener en cuenta que estas tipologías se potencian cuando hay factores de riesgo como el consumo de sustancias psicoactivas que, según la bibliografía y la evidencia, están presentes en los casos de $\boldsymbol{A S I}$ (Restrepo, 2007).

De acuerdo con lo anterior, si se desea evitar que el fenómeno del $\boldsymbol{A S I}$ se siga reproduciendo, resulta necesario un abordaje mucho más completo que considere, entre otras cosas, esas psicopatologías. Lo anterior urge en tanto esta problemática, en Colombia, alcanza proporciones preocupantes para el bienestar de la población infantil. Según reporta (Unicef, 2014) en el 2014 cerca de $\mathbf{1 0 , 4 4 2}$ presuntos casos de $\boldsymbol{A S I}$ ocurrieron a nivel nacional, siendo el género femenino el más afectado (INMLYCF, 2014)

Por consiguiente -y como se mencionó en la introducción- en el país se han desarrollado medios preventivos y de atención contra el abuso sexual infantil que se enfocan en las posibles víctimas (los menores). A través de estos los menores logran evitar ser víctimas o, de presentarse el caso, reciben atención psicológica y médica inmediata. Sin embargo, se carece de medios preventivos enfocados hacia los agresores y pedófilos para que estos no incurran en dicho comportamiento (Vélez, Henao, Ordoñez, \& Gómez, 2015; Te protejo, 2018; Castellanos, Olmos, \& Hernández, 2016; Goicoechea, Nañez, \& Molino, 2001; ICBF, 2012). Existe entonces un vacío en términos de intervención al carecer de un modelo amplio, profundo e integral contra el $\boldsymbol{A S I}$.

Como si fuera poco, también existe una gran confusión en la terminología que lleva a considerar pedófilo y pederasta, pedofilia y abuso como sinónimos cuando en realidad no lo son, Esta confusión tiene serias consecuencias en tanto repercute en las concepciones y representaciones que la sociedad tiene y además dificulta la creación de buenos programas de intervención dirigidos hacia esta población (Beier, et-al, 2009a; 2009b). Con fines aclaratorios, el termino pedofilia refiere a la existencia de un deseo y atracción sexuales hacia los niños(as), mientras que el término pederasta refiere a incurrir en comportamientos sexuales con menores (Arias \& Azor, 2009). Teniendo esto presente, la pedofilia y el $\boldsymbol{A S I}$ (en tanto abuso) son dos fenómenos diferentes, de modo que alguien con pedofilia, a pesar de tener una predisposición, no necesariamente abusará de un menor de edad.

Con el fin de encontrar un modelo que contribuya a llenar ese vacío que existe en Colombia, se buscó y encontró que en Alemania existe un programa con las características necesarias para suplir las necesidades mencionadas hasta el momento. Dicho programa es

el Proyecto Dunkelfeld, desarrollado en Berlín con el fin de atender potenciales agresores sexuales de menores brindándoles a pedófilos, quienes no hayan agredido sexualmente a un menor, la oportunidad de recibir tratamiento a sus inclinaciones sexuales desviadas en completo anonimato (Beier, et-al, 2009a). El tratamiento tiene un enfoque cognitivo-conductual y ha dado resultados con agresores sexuales ya capturados (Marshall, 2006, citado por Beier, et-al, 2009a).

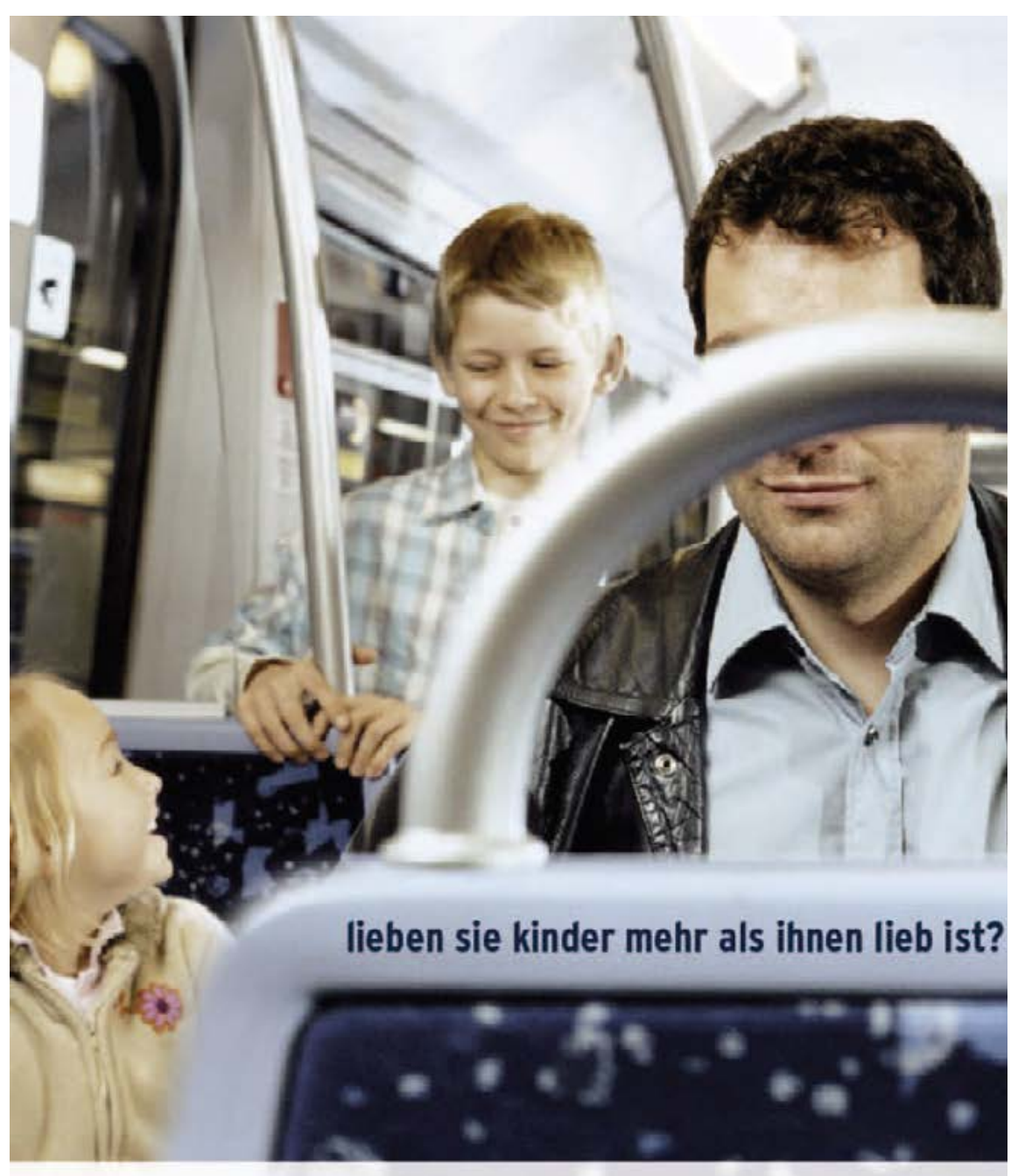

es gibt hilfe! kostenlos und unter schweigepflicht. institut für sexualmeđizin der charité, telefon: 030/450 529 450, www.kein-taeter-werden.de

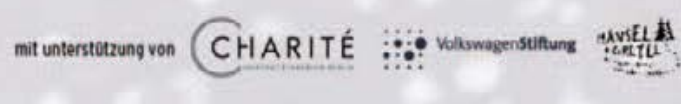

Imagen recuperada de (Beier, et-al, 2009a).

\section{Proyecto Dunkelfeld.}

El proyecto tiene dos fases. La primera es mediática y pretende llegar a los individuos con un interés sexual hacia menores de edad. La segunda busca llegar a sujetos con un fuerte deseo sexual hacia niños y esta campaña está orientada por individuos con estas parafilias. Así, algunos pedófilos se involucran y ayudan en la construcción de material mediático para generar mayor retentiva en esta población (Feelgood, Ahlers, Schaefer, \& Ferrier, 2002, citados por Beier, et-al, 2009a).

Esta estrategia mediática se mueve bajo cinco principios fundamentales (Beier, et-al, 2009a):

mostrar entendimiento y empatía hacia la población objetivo no realizar una campaña discriminante hacia las orientaciones sexuales de estos individuos reducir el temor a enfrentar penas por el sistema judicial asegurar confidencialidad y anonimato reducir sentimientos de culpa y vergüenza.-

En el afiche, la frase "Lieben sie kínder mehr als ihnen lieb ist?" se traduce a "¿Te gustan los niños más de lo que te gustaría?" 
Para lograr éste último punto (5) el siguiente mensaje es enviado "Tú no eres culpable de tus deseos sexuales, pero Tú eres responsable de tu comportamiento sexual ¡Hay ayuda! ¡No te conviertas en un agresor!" (Beier, et-al, 2009a, pág. 856)-.

Estos cinco (5) principios destacan el enfoque preventivo y mediante estos el proyecto busca alejarse de un movimiento de penalización, relacionándose con prácticas de criminología preventiva y no de justicia punitiva. Para la adecuada implementación de estos principios y lograr una campaña no discriminante.se cree necesaria la eliminación de pre-concepciones en torno a la pedofilia, pues no se desea generar una concepción errada del proyecto como una apología a la pedofilia.

Para la campaña se crearon posters y propagandas de TV que buscan ser medio de prevención con un alcance nacional. A continuación, se presenta un ejemplo (Beier, et-al, 2009a):

Paralelo a la campaña mediática se desarrolló una página web del proyecto como espacio para profundizar en la misma y donde la población objetivo puede hablar vía chat con profesionales. En la página también se ofrece información y educación al resto de la población alemana sobre el proyecto y sobre la pedofilia, con el fin de generar un impacto a gran escala (Beier, et-al, 2009a).

Por otro lado, se crearon oficinas con profesionales para que personas con atracción sexual hacia menores fueran a recibir la asistenciay las primeras evaluaciones. Para la primera fase del programa terapéutico fueron seleccionados individuos con las siguientes características: hombres quienes no hubiesen abusado de menores, pero quienes temieran incurrir en esas conductas; sujetos quienes han abusado de menores y temen el reincidir y además son desconocidos para el sistema legal; e individuos quienes han abusado de menores y temen reincidir, conocidos por el sistema legal pero quienes no son monitoreados por el mismo. De estos sujetos población objetivo $\mathbf{4 7 6}$ asistieron al programa y $\mathbf{2 4 1}$ lo terminaron (Beier, et-al, 2009a). Respecto al alcance del programa, para el 2007 (pasados los primeros 18 meses) se registró que hubo sujetos quienes se movilizaban más de 700 km con el objetivo de recibir el apoyo que éste brindaba.

Lo anterior indicó que potenciales agresores sexuales de menores sí pueden ser alcanzados por medio de una campaña mediática con fines de prevención primaria (Beier, et-al, 2009a; 2009b). Gran parte del éxito del programa se debe a su abordaje multidisciplinar pues son clave las relaciones entre profesionales de salud mental como psicólogos y psiquiatras, profesionales de relaciones públicas y las alertas mediáticas generales a la población contra el $\boldsymbol{A S I}$.

Tras hacer una revisión del programa, la principal conclusión formulada por (Beier, et-al, 2009a) fue que esta población sí puede ser alcanzada por una campaña mediática siempre y cuando, esta no tenga un enfoque de juicio moral respecto a las preferencias sexuales por menores de edad -es decir, siempre y cuando se aborde desde un enfoque que no esté guiado por prejuicios ni malas concepciones en torno a la pedofilia (McCartan, 2008; Niiler, 2011), en el que se haga claridad que se está hablando de un trastorno mental (Berlin, 2014) y que, por consiguiente, evite establecer la relación causal pedofilia-agresión sexual (McCartan, 2008)-. Por lo tanto, si se maneja correctamente el proyecto manteniendo al margen los prejuicios, el tratamiento puede brindarle a la población objetivo la oportunidad de controlar sus impulsos y la oportunidad de llevar una vida más acorde con las demandas sociales, previniendo de este modo el $\boldsymbol{A S I}$.

Ahora bien, ¿Por qué resulta importante este abordaje? Porque a pesar que estudios han indicado que no todos los pedófilos son agresores sexuales (Beier, et-al, 2009a; Arias \& Azor, 2009), en algunos sectores es una creencia popular que el hecho de ser pedófilo implica que la persona va a incurrir en $\boldsymbol{A S I}$. A este tipo de generalizaciones se les conoce como falacia de la falsa causa, la cual toma lugar cuando se produce una inducción incorrecta proponiendo una falsa causa para un evento, resultado o conclusión. Esto usualmente ocurre cuando un evento sucede inmediatamente antes que otro y el primero se asocia como causa del segundo, o también cuando se asume una situación de correlación tipo causa-efecto (Ibañez \& Torres, 1994; Custodio, 2009)

Una falacia similar es la generalización apresurada que se presenta cuando ocurre una conexión incorrecta entre eventos. Al tomar unos pocos datos o información insuficiente, se generaliza la conclusión sin tomar en cuenta otros factores que puedan influir en el efecto resultante (Ibañez \& Torres, 1994; Custodio, 2009). Un ejemplo de esta falacia es la siguiente genralización: "unos musulmanes han causado actos terroristas, (por ende) todos los musulmanes son terroristas".

No obstante, aún se cree la falacia que pedofilia es sinónimo de agresor sexual y tal situación genera un estigma social que causa aislamiento en personas que padecen el trastorno pedofilia. Lo anterior entorpece o evita la búsqueda de apoyo o la creación de medios de prevención terapéuticos enfocados hacia esta población. Evidencia de lo anterior es el hecho que a nivel mundial solo exista un centro preventivo no-carcelario para esta población (Beier, et-al, 2009a; 2009b)

Ante esta situación, las psicologías jurídica y forense pueden ayudar a eliminar las falacias en torno a esta situación y brindar medios que sirvan para el tratamiento de los pedófilos. Por un lado, las teorías desarrolladas sobre pedofilia y agresores sexuales permiten determinar las tipologías de agresores sexuales con el fin de lograr un alcance preventivo acorde a las condiciones psicológicas de los sujetos. Por otro lado, en convergencia con la psicología clínica se pueden desarrollar tratamientos preventivos ${ }^{2}$ para pedófilos que han y no han cometido $\boldsymbol{A S I}$.

Siguiendo esta línea, el Proyecto Dunkelfeld, con más de diez años en funcionamiento, se constituye una propuesta válida como medio adecuado de prevención integral de $\boldsymbol{A S I}$ en tanto contempla también a los agresoresy, en consonancia, formula planes quealcanzan a esta población. Éste ha sido un modelo exitoso que ha atraído a más de 8,500 personas en busca de ayuda y que ha sido reconocido por publicaciones académicas (Beier, et-al, 2009a; 2009b), importantes diarios del mundo e incluso por El Espectador en nuestro país (McGuinnes, 2015; Sánchez, 2015; Rubiano, 2017). Por lo anterior, a continuación se presenta una breve revisión de la viabilidad de su aplicación en Colombia, tomando en cuenta el contexto del país y todos los aportes que la psicología jurídica puede brindar para su óptimo desarrollo.

A propósito del siguiente apartado, resulta preciso hacer una anotación importante: teniendo en cuenta que la óptica desde la que se desarrolla el presente escrito y se fundamenta el proyecto viene de la psicología, en Colombia resulta necesario atender a la Ley 1090 de 2006 respecto a los dilemas deontológicos que puedan surgir en el curso de esta propuesta. De acuerdo con dicha ley y dentro del marco legal colombiano, el psicólogo no está bajo la obligación de romper el secreto profesional en caso tal que se le informe de un delito, especialmente considerando que el secreto profesional tiene protección constitucional y que el psicólogo está exonerado si reporta conductas punibles (Constitución Política de Colombia, 1991; Ley No 1090 , 2006). Lo anterior en tanto algunos de los participantes podrían ya haber agredido a menores con anterioridad o podrían estar en riesgo de agredir.

En el presente texto, el término preventivo se refiere al medio con el cual se evita la ocurrencia del abuso. Por lo tanto, si bien hay un factor de intervención terapéutica con la población objetivo, su foco principal es la prevención con los menores que pueden llegar a ser víctimas. 


\section{Viabilidad en Colombia}

Paralaformulación delaviabilidad teórica deun programaesimportante revisar el soporte empírico de uno de sus enfoques principales, para este caso específico: el tratamiento a pedófilos con el fin de disminuir sus riesgos de incurrir en $\boldsymbol{A S I}$ (Beier, et-al, 2009a; 2009b). Por ende, se deben revisar los programas de tratamiento actuales, la evidencia del éxito del apoyo mutuo entre la psicología y psiquiatría clínica, así como las evidencias estadísticas del éxito que los programas/tratamientos han tenido, para así determinar el tipo de terapias y abordajes necesarios para esta población en particular.

En su revisión, (Kear-Colwell \& Boer, 2000) plantean que, en algunos aspectos, la intervención a pedófilos no es del todo diferente a la de otros pacientes, incluso presentan las mismas resistencias al tratamiento y actitudes de confrontación con los terapeutas similares a las de todo tipo de paciente, sólo que reforzadas por los prejuicios sociales existentes en torno a esta parafilia. Esta resistencia es mayor si se le acusa de haber cometido un delito o de reincidir en $\boldsymbol{A S I}$.

El primer paso para una adecuada intervención -tanto en estos casos en particular como en cualquier proceso de intervención psicológica- consiste en que el terapeuta lleve a cabo un abordaje empático y de soporte. Sin embargo, en intervención con pedófilos el establecimiento del rapport tiene unas implicaciones más profundas apoyadas en la teoría del apego, pues esa resistencia y dificultad en la vinculación con otras personas -que supone un reto en el proceso de tratamiento- atiende a sus modelos de apego establecidos, principalmente a patrones de apego inseguro. En consonancia con lo planteado por la teoría se debe generar una aproximación de confianza con la población a tratar (Kear-Colwell \& Boer, 2000).

Otro requerimiento es evaluar la presencia de otros trastornos mentales, principalmente los de personalidad, pues no solo implican una reto adicional en el tratamiento sino que pueden estar correlacionados con comportamientos evitativos y defensivos en el ambiente terapéutico (Morrison, 2015) Sin embargo, a pesar de todas las dificultades y retos, sí puede existir un adecuado tratamiento a estas personas (Kear-Colwell \& Boer, 2000).

Como alternativas, existen terapias individuales de orden cognitivo-conductual enfocadas hacia programas de prevención en conjunto con otros tratamientos, las cuales generan resultados en situaciones de alto riesgo (Woodworth, et-al, 2013 citado por Balbuena, 2014). También hay soporte empírico sobre las ventajas que trae la terapia en grupo para los agresores sexuales en general y para los agresores de niños, niñas y adolescentes específicamente (Balbuena, 2014; Kear-Colwell \& Boer, 2000). De modo que ambos tipos de tratamiento (individual y grupal) pueden resultar exitosos para la población en cuestión.

El tratamiento con fármacos también ha sido considerado como un medio de intervención (Kear-Colwell \& Boer, 2000) en tanto se enfoca en reducir los impulsos, comportamientos y excitaciones sexuales desde una perspectiva orgánica en la que se recetan antiandrógenos y serotoninérigcos (Amelung, Kuhle, Konrad, Pauls \& Beier, 2012; Houts, Taller, Tucker \& Berlin, 2011 citados por Kear-Colwell \& Boer, 2000) y otros fármacos antilibidinales (Panesar, Allard \& Pai, 2011 citados por Kear-Colwell \& Boer, 2000). Esta aproximación destaca la importancia del trabajo en conjunto entre la psicología y la psiquiatría.

En este punto resulta importante referir a los planteamientos de (Balbuena, 2014), quien concluye que un adecuado tratamiento para la pedofilia requiere un abordaje interdisciplinar. En su revisión literaria encuentra la ausencia de tratamientos adecuados únicos que logren controlar, mitigar y eventualmente eliminar en su totalidad la condición particular de esa población, como el caso del uso de fármacos en conjunto con la terapia.

Desde la perspectiva comportamental también se ha intentado generar tratamiento para esta población mediante la terapia de aceptación y compromiso (ACT por sus siglas en inglés) que busca fortalecer el contexto adecuado del paciente para que evite sus comportamientos perjudiciales, tanto internos como externos (Díaz, 2016). Esta terapia se enfoca en la funcionalidad del sufrimiento psicológico ocasionado por la pedofilia y en los factores que soportan su mantenimiento, generando un análisis funcional del pedófilo y creando un tratamiento ideográfico. En cuanto al procedimiento, éste se desarrolla a partir de las conductas identificadas de la persona, por lo que el análisis histórico del paciente resulta fundamental -aspecto que guarda relación con otros métodos terapéuticos utilizados para esa población (Díaz, 2016; Balbuena, 2014; Kear-Colwell \& Boer, 2000)-. Es importante anotar que, si bien el análisis presentado por (Díaz, 2016) es de caso único, ya existe soporte teórico de la aplicación del análisis funcional del comportamiento en agresores sexuales (Fredericks \& Nishioka-Evans, 2000).

Los abordajes anteriormente mencionados indican que sí es posible realizar un tratamiento a los pedófilos, siempre y cuando sean abordajes amplios, incluyentes, profundos e integrales. En consonancia, desarrollar el programa en Colombia requerirá un abordaje multidisciplinar en el que, psicólogos y psiquiatras, lleven a cabo abordajes individuales y grupales, posiblemente empleando el uso de fármacos para unos casos. Dichos abordajes pueden hacerse desde un enfoque guiado por el análisis funcional del comportamiento, principalmente la terapia de aceptación y compromiso, o desde un enfoque cognitivo conductual, aunque esto no excluye otros modelos de psicoterapia que puedan resultar efectivos.

Es preciso tener en cuenta que, en últimas, las condiciones técnico-científicas están supeditadas a la voluntad política frente al fenómeno del $\boldsymbol{A S I}$ que tiene diversas aristas. Llevar a cabo un proyecto con un objetivo como el que plantea el Dunkelfeld supone enfrentarse a la opinión pública que, al calor de los hechos viralizados por los medios de comunicación y las redes sociales, tiende a buscar penas más severas contra los presuntos victimarios. Por lo tanto, buscar implementar un proyecto de este tipo requiere no sólo de ciertas condiciones técnico-científicas en el trabajo de campo sino que requiere también apoyo desde la educación en la academia, las instituciones públicas y la sociedad en general. Como se ha reiterado en varias partes del texto, un aspecto importante para el tratamiento de la pedofilia es la reducción de estigma y actitudes punitivas hacia personas con esta condición, pues una reducción del estigma hacia los pedófilos no solo facilita que ellos busquen apoyo sino que genera una actitud terapéutica en la población que facilita las intervenciones y abre paso a la intervención en conjunto con sujetos que padecen el trastorno (Harper, Bartels, \& Hogue, 2016)

Lo anterior resulta fundamental pues si se revisan los proyectos de ley que han cursado en el congreso en la última década, el carácter preventivo se reduce a penas superiores sustentadas en razones mediáticas y de beneficios electorales las cuales están pensadas a corto plazo y no resultan ser efectivas, tal cual lo ha señalado la (Comision Asesora de Politica Criminal (2012). En consonancia, si se revisan en detalle el Proyecto de Ley N 041, 2016 es posible evidenciar un errado abordaje del concepto de pedofilia que, por consiguiente, tiene implicaciones en el imaginario colectivo. De ahí que resulte importante que el trabajo de los profesionales tenga como punto de partida la educación frente a la salud mental y las psicopatologías como la pedofilia, lo cual se logra mediante el apoyo de los medios de 
comunicación y la academia, así como de la participación activa en la construcción de políticas públicas, asunto que a veces los psicólogos y psiquiatras ven ajeno a su quehacer.

A pesar de los múltiples retos, el proyecto es una empresa válida y necesaria en Colombia; según la revista (Semana, 2016) se estima que hay alrededor de $\mathbf{4 8 0 , 0 0 0}$ pedófilos en el país, investigaciones como la de (Posada \& Salazar, 2005) y el trabajo de (Trabazo \& Azor, 2009) dan cuenta que sujetos quienes han cometido abuso sexual de menores padecen de esta parafilia, además de otros trastornos psicológicos. Lo anterior lleva a que se considere la propuesta de (Kaplan, 2015) sobre el abordaje de la pedofilia dentro de los marcos jurídicos, la cual invita a concebir el fenómeno más allá de una categoría jurídica -como se ha concebido-e incluirla como una cuestión de salud pública que requiere ser atendida y requiere ser incluida dentro de los programas de prevención en salud mental, en tanto los potenciales victimarios también son sujetos de derecho.

\section{Aliados - Los Medios}

Ahora bien, uno de los aspectosfundamentales del Proyecto Dunkelfeld es el uso de medios de comunicación para lograr abarcar el territorio nacional y así atraer pedófilos de toda Alemania para su tratamiento (Beier, et-al, 2009a). Para una adecuada aplicación de este proyecto en Colombia se deben entonces considerar los medios de divulgación, los mensajes a dirigir y las instituciones que puedan ayudar en el proyecto tanto para fines divulgativos como económicos. También se deben explorar las demandas de cada ambiente en términos de la divulgación, y considerar la gran variación geográfica y tecnológica del país.

Colombia, actualmente tiene una gran variedad de medios de comunicación masiva. La televisión es uno de los que mayor alcance tiene a nivel nacional (Correa \& Armando, 2012), medios más recientes e innovadores como el internet y las redes sociales permiten una mayor difusión en tanto propagan mensajes con mayor alcance y facilidad (Parada, 2013). Cuando se habla de un medio de comunicación masiva se refiere a mensajes que son enviados por un emisor y recibidos de forma idéntica por una gran variedad de receptores; los medios más conocidos y utilizados son la televisión, la radio y el periódico (Goya, 2012) Tomando en cuenta los presupuestos del Proyecto Dunkelfeld (Beier, et-al, 2009a) el medio utilizado para divulgar los mensajes en Colombia debe ser de alto impacto y gran cobertura, buscando difundir el mismo mensaje a toda la población.

Los medios de comunicación masiva se clasifican como (Goya, 2012):

$\rightarrow$ primarios o propios, que están ligados al cuerpo y no requieren de tecnología para su comunicación; estos se producen cuando hay un orador, una presentación de una obra de teatro o un sermón y los receptores deben ser más de siete personas para considerarlo masivo

$\rightarrow$ secundarios, que requieren apoyo de tecnologías para su transmisión pero no para su recepción; estos incluyen revistas, periódicos, folletos, vallas, entre otros

$\rightarrow$ terciarios, que requieren tecnología para su transmisión y recepción; aquí destacan la televisión y la radio

$\rightarrow$ cuaternarios o medios digitales, son las nuevas tecnologías de comunicación que permiten comunicación bilateral; el internet y las redes sociales son sus mayores representantes.

Para la adaptación del Proyecto Dunkelfeld en Colombia, los medios principales a utilizar serían los secundarios, terciarios y cuaternarios, puesto que tienen mayor alcance. Su adaptación requeriría del apoyo de instituciones como el ICBF, Ministerio de Salud, Fiscalía y Medicina Legal, entre otros medios de orden público y privado, que apoyen la difusión nacional del mensaje. Tomando en cuenta los costos que traería tal adaptación y eventual implementación, sería necesario presentar a estas entidades una propuesta sobre el proyecto y su necesidad de difusión.

El ideal sería contar con el apoyo de los medios de mayor alcance en sus respectivas categorías, como los canales de televisión RCN y Caracol, los más vistos a nivel nacional (Ipsos, 2008), así como las diferentes emisoras colombianas, con el fin de difundir en diferentes horarios. En términos del alcance, debe alcanzar y abarcar todas las clases y estratos sociales, puesto que el trastorno de pedofilia o la predisposición a cometer un abuso sexual infantil (Arias \& Azor, 2009; Echeburúa \& Guerricaechevarría, 2000; Kaplan, 2015) puede sufrirlo cualquier persona en cualquier contexto.

Tal cual sucede en Alemania, la población objetivo principal (o primaria) serían los pedófilos, y la secundaria sería el resto de la población. El movimiento mediático dirigido a la población principal sería similar al del Proyecto Dunkelfeld original, estaría guiado bajo los mismos cinco (5) principios y emitiría mensajes similares en los que se invite a buscar apoyo y no se culpabilice. La divulgación se llevaría a cabo mediante posters y por el medio televisivo, en apoyo conjunto con las redes sociales.

La población secundaria también es de gran relevancia, puesto que Colombia es un país cuya gran parte de su población tiene valores conservadores y, debido a esto el mensaje posible y fácilmente puede ser mal-interpretado como una apología al pederasta o a la pedofilia. Para evitar lo anterior, se considera prudente, al menos inicialmente, difundir mensajes educativos por los mismos medios, los cuales den a entender que no se está realizando una apología al abuso sexual infantil, sino una propuesta de apoyo que puede reducir este tipo de agresiones. Por tanto, respecto a la población secundaria resulta importante diferenciar entre pedófilo y pederasta (Arias \& Azor, 2009), aun cuando la intervención vaya enfocada a las dos.

Por último, es importante evitar que los mensajes dirigidos a la población secundaria no afecten aquellos dirigidos a la población primaria, sino que por el contrario complementen. Así pues, lo que se divulgue de manera educativa no debe afectar los cinco (5) principios y no debe opacar o desbordar los medios. La difusión de la información educativa a esta población secundaria puede ser por medio de una página web.

En lo que a medios refiere, éstos serían los aspectos a considerar para la adaptación del Proyecto Dunkelfeld en Colombia. Cabe recalcar que la viabilidad no asegura su éxito, aun cuando la necesidad por un programa de esta índole existe y es evidente. Dado que el reto es de grandes proporciones, a la hora de implementar esta propuesta (Rodríguez, 2003) será clave el trabajo interdisciplinar entre psicólogos, psiquiatras, abogados, comunicadores, entre otros. En el caso de la psicología -en tanto ciencia sobre la que se apoya esta iniciativa- los retos que ésta debe asumir incluyen incidir en la cultura y desarrollar el proceso investigativo, que debe ser constante para dar linealidad y coherencia al proyecto en Colombia.

Entre los tantos saberes que aporta la psicología está el hecho que hay personas quienes no presentan factores de riesgo evidentes, pero sí hay aspectos de personalidad o patrones culturales que les impulsa a incurrir en agresiones sexuales infantiles. Ante esta situación, resulta necesario que la campaña no sólo se dé en las zonas que presentan mayores factores de riesgo sino en todo el territorio nacional, pero que en estas áreas de mayor riesgo se haga más fuerte la campaña mediática. 


\section{Proyecto Dunkelfeld en Colombia}

\section{Viabilidad teórica}

Además de brindar guía a la campaña, desde la psicología jurídica, forense y clínica se puede hacer investigación con los pedófilos quienes atiendan a la prevención primaria. Manejando siempre el anonimato, la información obtenida puede ser empleada como fuente de investigación, en pro de mejorar las teorías existentes sobre la pedofilia y, por ende, optimizando así los abordajes psicológicos forenses en casos de presunto abuso sexual infantil. El proceso para las investigaciones sería el siguiente:

la persona accede al centro de prevención primaria firma un consentimiento informado donde acepta que la información brindada podrá ser usada para investigaciones psicológicas, si y sólo si hay anonimato en el manejo de la información

la información obtenida de las personas tratadas es sistematizada

a partir de la información sistematizada se desarrolla la investigación.

De manera paralela, la psicología jurídica se apoyaría tanto en la campaña dirigida a la población objetivo como en aquella de sensibilización de la población secundaria, con el fin de asimilar y trabajar la diferencia entre pedófilo y pederasta -(el primero tiene una parafilia (APA, 2014)) y es una persona con una atracción sexual por niños, mientras que el segundo es quien comete una agresión sexual hacia un menor de edad (Arias \& Azor, 2009). En la campaña de sensibilización, la psicología jurídica podría aportar los parámetros o los discursos mediante los cuales se enseñaría a la población esta diferencia. En la campaña guiada por psicólogos jurídicos se mostraría la pedofilia como un trastorno o enfermedad mental (APA, 2014) que requiere tratamiento y apoyo más que rechazo social, y en la campaña dirigida a la población objetivo se transmitiría de manera clara que no se trata de una apología a la pederastia.

Aunque lo abordado hasta el momento deja más que clara la importancia e incidencia de campos y disciplinas tales como la comunicación social, el periodismo, la publicidad, entre otras afines, también queda claro que esta área de la comunicación masiva ha sido poco explorada por la psicología jurídica y forense en Colombia. Este señalamiento es fundamental pues en un país como Colombia la comunicación masiva tiene una importante influencia en la percepción de seguridad, en la movilización social, en la construcción de políticas públicas y, por consiguiente, en el quehacer de los psicólogos quienes trabajan en el campo del derecho.

Por lo tanto, pensar en aliados, como los medios, es también pensar en un campo fértil sobre el que se debe contribuir desde el saber psicológico para la construcción de una cultura de salud mental que facilite la prevención de delitos, no sólo el $\boldsymbol{A S I}$ sino todos aquellos asociados a un malestar en la psique.

\section{Conclusiones}

A partir de la revisión realizada se pueden formular una serie de conclusiones y consideraciones para la aplicación o desarrollo del Proyecto Dunkelfeld en Colombia (o una propuesta similar). La primera es que teóricamente la propuesta resulta viable en tanto se ha demostrado que la población objetivo puede recibir tratamiento y Colombia cuenta con los medios de comunicación para llevar a cabo una campaña mediática a nivel nacional. Sin embargo, para hacer esto se necesitaría del apoyo de profesionales clínicos de la salud en el área de tratamiento (como psicólogos y psiquiatras) y una gran inversión económica y apoyo estatal para la divulgación del proyecto en todo el país.
Consecuentemente uno de los primeros pasos a seguir sería hacer una investigación sobre los costos (económicos, científico-académicos y sociales) que implicaría diseñar e implementar el proyecto. En esta revisión de costos es necesario determinar el presupuesto base que contempla la construcción de la página web, la divulgación mediática, la contratación de cierta cantidad de profesionales, las investigaciones académicas a realizar y las instalaciones donde se llevaría a cabo el tratamiento. Asimismo, se considera prudente investigar cuáles instituciones pueden apoyar en la construcción del proyecto tanto para su financiación como su divulgación. De igual manera, se deben estimar las posibles resistencias que haya dentro del imaginario colectivo que implicarán una inversión extra en medios y profesionales para construir un espacio más abierto a la propuesta.

Otro aspecto importante a considerar es el contexto cultural en Colombia que da pie a confusiones en cuanto a la percepción de la propaganda, al confundirla con una apología a la pedofilia o al ASI. Desconocer este contexto puede comprometer todo el proyecto, pues podría no haber una adecuada recepción del mismo, lo cual podría llevar a su cierre. Un ejemplo de lo anterior es la polémica generada por las cartillas de diversidad sexual que intentó difundir el Ministerio de Educación (Redaccion el Herlado, 2016)

Ante esta situación se considera necesario que la primera campaña se enfoque en la población secundaria (no los pedófilos sino los colombianos en general), buscando enseñar sobre la importancia de la prevención en esta población, diferenciando pedofilia de pederastia y mostrando cómo este abordaje puede llevar a evitar que niños, niñas o adolescentes sean víctimas de abuso sexual. Esto se debe organizar de forma tal que esta campaña no sea contradictoria sino complementaria a aquella enfocada a la población primaria (los pedófilos).

Respecto al apoyo de la psicología jurídica y forense en el proyecto, éste se daría en dos aspectos fundamentales: el primero es la construcción de la campaña para ambas poblaciones, buscando educar a la secundaria y alcanzar a la primaria. El segundo es apoyando las investigaciones y brindando soportes científicos sobre los lugares o regiones de Colombia que requerirían un mayor apoyo y una mayor divulgación mediática, lo anterior mediante el estudio de las teorías existentes sobre factores de riesgo que pueden brindar suficiente información al respecto y que apoyen la implementación de la campaña. De esta y otras maneras los psicólogos forenses y jurídicos serían una parte integral en la adaptación del proyecto.

Complementario a todo lo anterior, resulta necesario desde la academia hacer meta-análisis que permitan identificar los mejores medios de tratamiento para la población primaria, sí como estudios empíricos que puedan reforzar dichos tratamientos.

\section{Conflicto de interés}

Los autores declaramos no tener ningún conflicto de interés.

\section{Agradecimientos}

Los autores agradecen a Ángela Tapias por su revisión y aportes al primer borrador de este texto. 


\section{Referencias}

APA. (2014). Desk reference to the diagnostic criteria from DSM-5. Washington, DC. APA: American Psychiatric Publishing. Recuperado de https://www.appi. org/Desk Reference to the Diagnostic Criteria From DSM-5

Arias, V \& Azor, L. (2009). La pedofilia: un problema clínico, legal y social. Edu Psykhé. 8(2): 195-219. Recuperado de https://dialnet.unirioja.es/servlet/ articulo?codigo $=3145999$

Balbuena, F. (2014). Cartografiando la pedofilia: eficacia de los tratamientos y estrategias futuras. Apuntes de psicología. 3. 245-250. Recuperado de http://www.apuntesdepsicologia.es/index.php/revista/article/view/522

Becerra-García, J. \& García-León, A. (2012). Trastorno mental en los agresores sexuales: estudio descriptivo de muestra española. Revista española de medicina legal. 39(1): 3-6. DOI: 10.1016/j.reml.2012.10.007

Beier, K., Neutze, J., Munndt I., Ahlers, C., Goecker, D., Konrad, A. \& Schaefer. (2009b). Encouraging self-identified pedophiles and hebephiles to seek proffesional help: First results of the Prevention project Dunkelfeld (PPD). Child abuse \& Neglect. 33: 545-549. DOI: 10.1016/j.chiabu.2009.04.002

Beier, K.M., Ahlers, C.J., Goecker, D., Neutze, J., Mundt, I.A., Hupp, E. \& Schaefer, G.A. (2009a). Can pedophiles be reached for primary prevention of child sexual abuse? First results of the Berlin prevention project Dunkelfeld (PPD). The journal of forensic psychiatry \& psychology. 20(6); 851-867. DOI: 10.1080/14789940903174188.

Berlin, F. (2014). Pedophilia and DSM-5: The importance of clearly defining the nature of a pedophilic disorder. The journal of the American Academy of Psychiatry and the law. 42: 404-407. Recuperado de http://citeseerx.ist.psu. edu/viewdoc/download?doi=10.1.1.881.9053\&rep=rep1\&type=pdf

Bieber, E. (2012). Perfil psicosocial del pedófilo. Alcmeon, revista argentina de clínica neuropsiquiatríca. 17(3): 268-276. Recuperado de https://www. alcmeon.com.ar/17/67/08_bieber.pdf

Bienestar Familiar. (2012). Protegiendo a la niñez de la violencia sexual. Observatorio del bienestar de la niñez. Colombia. Recuperado de http://www.icbf.gov.co/portal/page/portal/Observatoriol/Archivo/2012/ publicacion-25.pdf

Britto, X. (2009). La congestión judicial en Colombia. Trabajo de grado. Pontificia Universidad Javeriana. Facultad de comunicación y lenguaje. Recuperado http://www.javeriana.edu.co/biblos/tesis/comunicacion/tesis202.pdf

Calderón, L. (2016). Ensayo: Congestión Judicial en la jurisdicción de lo contencioso administrativo Colombiana. Revista Dikaión- Lo justo. (11) 58-71

Caracol Radio (2011). En Colombia hay más de dos millones de procesos represados. Caracol radio. Recuperado de http://caracol.com.co/ radio/2011/09/08/hacional/1315473540 544242.html

Castellanos, D., Olmos, A. \& Hernández, D. (2016). Estrategias de prevención abuso sexual infantil. Corporación Universitaria Minuto de Dios. Programa Licenciatura en Pedagogía infantil. Bogotá; Colombia. Recuperado de http://repository.uniminuto.edu:8080/xmlui/bitstream/handle/10656/4543/ TPED MoraCastellanosDianaCarolina 2016.pdf? sequence=1

Comisión asesora de política criminal (2012). Diagnóstico y propuesta de lineamientos de política criminal para el estado colombiano. Informe final. Bogotá: ministerio de justicia y del derecho. Recuperado de https:// www.minjusticia.gov.co/Portals/0/INFO\%20POLI\%20CRIMINAL_FINAL23NOV. $p d f$

Constitución Política de Colombia (1991). Asamblea Nacional Constituyente (7 de julio de 1991). [Artículo 74 - Titulo II]. 2da Ed. Legis Recuperado de http:// www.corteconstitucional.gov.co/inicio/Constitucion\%20politica\%20de\%20 Colombia.pdf

Correa, M \& Armando D. (2012). Medios de comunicación en Colombia. Treinta años de Investigación y reflexión. Nómadas (Col). 36: 271-273. Recuperado de http://www.redalyc.org/pdf/1051/105124264019.pdf

Custodio, S. (2009). Introducción a la lógica. Editorial Oscar de León Palacios. Recuperado de https://nonosky.files.wordpress.com/2010/02/introduccion-ala-logica-sergio-custodio.pdf

Díaz, J. (2016). (2016). Tratamiento de un caso de pedofilia desde la terapia de aceptación y compromiso (ACT). Avances en Psicología Latinoamericana. 34(3), 529-541. Recuperado de http://revistas.urosario.edu.co/index.php/apl/ article/view/2881

Echeburúa, E. \& Guerricaechevarría, C. (2000). Abuso sexual en la infancia: víctimas y agresores. Un enfoque clínico. Barcelona: Ariel.
Fredericks, J. \& Nishioka-Evans, V. (2000). Evaluación funcional de una población de agresores sexuales. Reep \& Horner (Ed.). Análisis funcional de problemas de la conducta, Madrid: España. Paraninfo Thomson Learning.

Goicoechea, P., Nañez, A. \& Molino, C. (2001). Abuso sexual infantil: Manual de formación para profesionales. Primera Edición. Save the children. Recuperado de https://www.savethechildren.es/sites/default/files/imce/ docs/manual abuso sexual.pdf

González, J. \& González, S. (2004). Evaluación del video "el árbol de chicoca” para la prevención del abuso sexual infantil en el caribe colombiano. Revista psicogente. 12: 60-69 Recuperado http://revistas.unisimon.edu.co/index. php/psicogente/article/view/1112

Goya, E. (2012). Medio de comunicación masiva. Red tercer milenio. México. Recuperado de http://www.aliat.org.mx/BibliotecasDigitales/comunicacion/ Medios de comunicacion masiva.pdf

Harper, C., Bartels, R \& Hogue, T. (2016). Reducing stigma and punitive attitudes toward pedophiles through narrative humanization. A journal of sexual abuse. 1-23. DOI: 10.1177/1079063216681561.

Ibañez, A.H. \& Torres, J.A. (1994). Falacias. México: Editorial Torres Asociados. Recuperado de https://www.elsotano.com/libro-falacias-10105769.

INMLYCF. (2016). Forensis: Datos para la vida. INMLYCF. 18(1). Recuperado de http://WwW.medicinalegal.gov.co/documents/20143/49526/ Forensis+2016.+Datos+para+la+vida.pdf

INMLYCF. (2017). Violencia contra las mujeres: Colombia, comparativo años 20162017 (enero a octubre). INMLYCF. Recuperado de http://WWW.medicinalegal. gov.co/documents/20143/57992/Violencia+contra+las+mujeres.pdf

INMLYCF. (2014). Forensis Datos para la Vida: Herramienta para la Interpretación, Intervención y Prevención de Lesiones de Causa Externa en Colombia. INMLYCF. 16 (1): 181 - 279. Recuperado de http://wWW.medicinalegal.gov.co/ documents/20143/49520/Forensis+2014+Datos+para+la+vida.pdf

Ipsos. (2008). La gran encuesta de televisión nacional en Colombia. Bogotá, Colombia. Recuperado de http://static.iris.net.co/semana/upload/ documents/Doc-1774 20081024.pdf

Kaplan, M. (2015). Taking pedophilia seriously. Washington \& Lee Law Review. 72 (1), 75-170. Recuperado de https://scholarlycommons. law.wlu.edu/cgi/viewcontent.cgi?referer=https://scholar.google. es/\&httpsredir $=1 \&$ article $=4438 \&$ context $=$ wlult

Kear-Colwell, J. \& Boer, D (2000). The treatment of pedophiles: clinical experiences and the implications of recent research. International journal of offender therapy and comparative criminology. 44(5): 593-605. DOI:10.1177/0306624X00445006

Ley 1090 de 2006, (2006). Por la cual se reglamenta el ejercicio de la profesión de Psicología, se dicta el Código Deontológico y Bioético y otras disposiciones. Congreso de Colombia (6 de septiembre de 2006). Recuperado de http://colpsic.org.co/aym image/files/LEY 1090 DE 2006. $p d f$

Marroquín, N. (2016). Aplicación e implementación de la entrevista forense a menores de edad víctima de delitos sexuales en el circuito judicial de Zipaquirá, Cundinamarca. Facultad de Derecho. Universidad Militar Nueva Granada. Bogotá DC. Recuperado de http://repository.unimilitar.edu. co/bitstream/10654/15786/1/Marroqu\%C3\%ACnCabreraNancyStella2016.pdf

McCartan, K. (2008) Current understandings of paedophilia and the resulting crisis in modern society. In: Caroll, J. M. and Alena, M. K., eds. (2008) Psychological Sexual Dysfunctions. New York: Nova Biomedical, pp. 51-84 ISBN 9781604560480. Recuperado de http://eprints.uwe.ac.uk/13608

McGuinnes, D. (2015). El radical programa de Alemania para controlar el deseo de los pedófilos. BBC Mundo. Recuperado de http://www.bbc.com/mundo/ noticias/2015/07/150713 salud alemania pederastas terapia il

Ministerio de salud. (2015). Encuesta nacional de salud mental, tomo 1. Ministerio de Salud \& Colciencias. República de Colombia. Recuperado de https:// www.minsalud.gov.co/sites/rid/Lists/BibliotecaDigital/RIDE/DE/presentacionencuesta-nacional-salud-mental-2015.pdf

Morrison, J. (2015). DSM- $5^{\circledR}$ Guía para el diagnóstico clínico. Ciudad de México, México. El Manual Moderno.

Niiler, E. (2011). Siz misconceptions about pedophiles. Discovery news. Recuperado de http://www.unh.edu/ccrc/news/NiilerSixMisconceptionsAboutPedophiles.pdf

Parada, M. (2013, abril 15). RCN Radio está enfocada en su crecimiento en internet La República. Recuperado de http://wWw.larepublica.co/empresas/rcnradio-est\%C3\%A1-enfocada-en-sucrecimiento-en-internet 36287 


\section{Proyecto Dunkelfeld en Colombia}

\section{Viabilidad teórica}

Posada, E. \& Salazar, J. (2005). Aproximaciones criminológicas y de la personalidad del abusador sexual (tesis de pregrado). Universidad Católica de Oriente. Rionegro-Antioquía, Colombia. Recuperado de https:// s3.amazonaws.com/academia.edu.documents/31437650/perfil psicologico colombia.pdf?AWSAccessKeyId=AKIAIWOWYYGZ2Y53UL3A\&Expires=152220 7434\&Signature $=n 4$ DVXiguxQoGbsP4yh15mYSJdl0\%3D\&response-contentdisposition=inline\%3B\%20filename\%3Dperrfil.pdf

Proyecto de Ley No 041 (2016). Por medio del cual se crea el registro nacional de abusadores para la protección de los menores de edad. Bogotá. Colombia, Congreso de Colombia. Recuperado de http://wWw. comisionprimerasenado.com/documentos-pendientes-de-publicacion/ ponencias-y-textos-aprobados/1501-ponencia-primer-debate-p-l-80-de-2017senado-041-de-2016-camara/file

Redacción el Heraldo. (10 de Agosto 2016). Cinco puntos para entender la polémica con el Ministerio de Educación. El Heraldo. Recuperado el 27 de julio de 2017 de https://www.elheraldo.co/nacional/cinco-puntos-paraentender-la-polemica-con-el-nuevo-manual-de-educacion-sexual-escolar

Restrepo, E. (2007). Factores de riesgo y protección en los agresores sexuales infantiles. Revista skopein. 2: 40-45. Recuperado de http://skopein.org/ojs/ index.php/1/article/view/9/6

Rodríguez, L. (2003). Intervención interdisciplinaria en casos de abuso sexual infantil. Universitas Psychologica. 2(1):57-70. Recuperado de http://sparta. javeriana.edu.co/psicologia/publicaciones/actualizarrevista/archivos/ v2N107intervencion.pdf

Rubiano, M. (2017). "Cadena perpetua no evita delitos contra niños”: Penalista Iván González. El Espectador. Recuperado de https://www.elespectador.com noticias/judicial/cadena-perpetua-no-evita-delitos-contra-ninos-penalistaarticulo-674864

Sánchez, R. (2015). Una página web para el "autocontrol” de los pedófilos. El Mundo. Recuperado de http://www.elmundo.es/ espana/2015/07/15/55a558b3e2704e803a8b4582.html
Semana (2016). "En Colombia hay 480.000 pedófilos y la pregunta es dónde están?”. Recuperado de http://www.semana.com/vida-moderna/articulo/ como-se-desarrolla-la-pedofilia-y-pedarastia/508368

Te protejo (2018). Video de te protejo. Te protejo. Recuperado de http://wWw. teprotejo.org/index.php/es/quienesomos/videos-de-presentacion-de-teprotejo

Trabazo, V. \& Azor, F. (2009). La pedofilia: Un problema clínico, leal y social. eduPsykhé. 8(2): 195-219. Recuperado de http://repositorio.ucjc.edu/ bitstream/handle/20.500.12020/15/C00041835.pdf?sequence=1\&isAllowed=y

Unicef. (2014). Informe Anual. Fondo de las Naciones Unidas para la infancia (Unicef). Recuperado de http://unicef.org.co/documentos/INFORME ESP 190315.pdf

Unicef. (2017). Abuso sexual contra niños, niñas y adolescentes: Una guía para tomar acciones y proteger sus derechos. Fondo de las Naciones Unidas para la infancia (Unicef). Recuperado de https://wWw.unicef.org/argentina/ spanish/proteccion-AbusoSexual contra_NNyA-2016.pdf

Uribe, N. (2011). Abuso sexual infantil y administración de justicia en Colombia. Reflexiones desde la Psicología Clínica y Forense. Pensamiento psicológico. 9 (2): 183-202. Recuperado de http://wWw.scielo.org.co/scielo. php?script=sci arttext\&pid=S1657-89612011000100013

Vélez, C., Henao, P., Ordoñez, F \& Gómez, L. (2015). Evaluación de un programa de promoción de conductas de Autoprotección para la prevención del abuso sexual infantil, Medellín, Colombia. Revista Médica de Risaralda. 21(1):3-8. DOI: https://doi.org/10.22517/25395203.8855.

Villanueva, S. (2005). Factores protectores en la prevención del abuso sexual infantil. Liberabit. 11: 19-24. Recuperado de https://biblat.unam.mx/es/ revista/liberabit/articulo/factores-protectores-en-la-prevencion-del-abusosexual-infantil

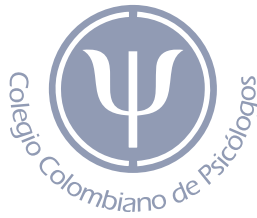

2)Ascofapsi

Asociación Colombiana de Facultades de Psicología
IBEROAMERICANA

CORPORACIÓN UNIVERSITARIA 